\title{
Rayleigh-Benard-Marangoni Ferro Convection with Concentration and Temperature Dependent Viscosity
}

\author{
V Kavitha, M Chenna Krishna Reddy, S Harisingh Naik
}

\begin{abstract}
The inception of Rayleigh-Benard-Marangoni (RBM) Ferro convection with concentration and temperature dependent viscosity is investigated theoretically and the resultant is further enhanced numerically using Galerkin method. We observed that the effect of Rayleigh number together with internal heating suppressed the onset of RBM Ferro convection. The nonlinear nature of magnetic fluid parameter has no impact on the onset of Ferro convection. The latent values found numerically by Galerkin weighted Residual technique and regular perturbation technique are found to be alike, indicating the fact that the obtained solutions are near exact in nature. The result of the BC's for lower and upper free rigid boundary at temperature dependent surface tension forces are found to be immaculately insulate to temperature perturbation.
\end{abstract}

Keywords: Rayleigh-Benard-Marangoni Ferro convection, Galerkin Method, Regular perturbation technique, internal heating.

\section{INTRODUCTION}

One of the instabilities studied comprehensively by many researchers nowadays is the inception of thermal convection in a thin horizontal layer of fluid insulated from below. Pearson [1] has considered surface tension to study Benard convection, whereas Lord Rayleigh [2] took convection through buoyancy. Here, we study buoyancy driven Rayleigh-Benard Convection with variable viscosity. Benard observed that instability arises, when a layer of the fluid resolves around the cells and was successful in photographing these cells. The experimental work of Benard was first investigated theoretically by Rayleigh and it is known as Rayleigh-Benard convection. The observations of cells carried out by Benard were thoroughly explained by Rayliegh through buoyancy. Nanjundappa et al.[4,5,6,7,8,9] found an exact solution for the through Marangoni-Benard ferro-convection with temperature dependent viscosity

Revised Manuscript Received on July 22, 2019.

V Kavitha, Department of $\mathrm{H} \& \mathrm{~S}$,Vardhaman College of Engineering, Hyderabad

Dr M Chenna Krishna Reddy, Department of

Mathematics,University College of Science, Osmania

University,Hyderabad

Dr S Harisingh Naik, Department of Mathematics,

University College of Science, OsmaniaUniversity,

Hyderabad
The fluid flow with uniform material properties is governed by Navier-Stoke's equation and the two non dimensional numbers, explicitly, the Rayleigh number $\left(R_{e}\right)$ and the Prandtl number $\left(P_{r}\right)$. Rayleigh showed that the stability of fluid layer heated from below depends on the numerical value of the non-dimensional number, $\mathrm{R}$ known as Rayleigh number. Rayleigh in turn observed that instability leaves, if $R \rightarrow R_{C} \quad, \quad R_{C} \quad$ indicates critical Rayleigh number, $\mathrm{R}=\mathrm{R}_{\mathrm{c}}$. The $P_{r}$ approximates kinematic viscosity in correlation with thermal diffusivity. In heat transfer problems, the Prandtl number controls the relative thickness of the momentum and thermal boundary layers. When $\operatorname{Pr}$ is small, thermal diffusivity is dominates, and the heat diffuses swiftly compared to the velocity (momentum). This means that for liquid metals the thickness of the thermal boundary layer is much superior to the velocity boundary layer.

There are fluids like glycerol, silicon oil and polybutene oil for which the viscosity varies strongly with temperature. To study the heat transfer precisely, variation in viscosity with temperature is to be considered. Viscosity depends exponentially upon the temperature is assumed. Since viscosity of a fluid decreases with increasing temperature therefore it influences the heat transport rates. Variation in viscosity has been considered to measure the accurate rate of heat transfer. Convection is the way of transfer of heat in which there is movement of the liquid molecules.

\section{MATHEMATICAL FORMULATION}

Convective motion in a horizontal fluid layer insulated from below is described by the continuity equation, the momentum equation and the heat equation.

The governing equations for the fluid layer are:

The continuity equation is

$\nabla \cdot \vec{V}=0$

The momentum equation

$\rho_{0}\left[\frac{\partial \vec{V}}{\partial t}+(\vec{V} \cdot \nabla) \vec{V}\right]=-\nabla p+\mu \nabla^{2} \vec{V}$

and the heat equation is

$\left[\frac{\partial}{\partial t}+\vec{V} \cdot \nabla\right] \theta=\kappa \nabla^{2} \theta$

The governing equations for Porous medium are :

The continuity equation is 
$\nabla_{n} \cdot \overrightarrow{V_{n}}=0$

The momentum equation

$\frac{\rho_{0}}{\phi} \cdot \frac{\partial \overrightarrow{V_{n}}}{\partial t}=-\nabla_{n} p_{n}-\frac{\mu}{\kappa} V_{0}$

and the heat equation is

$S \frac{\partial \theta_{n}}{\partial t}+\left(\overrightarrow{V_{n}} \cdot \nabla_{n}\right) \theta_{n}=\kappa_{n} \nabla_{n}^{2} \theta_{n}$

In the above equations, $\vec{V}=(u, v, w)$ is the velocity vector, $\theta$ is the temperature , $p$ is the pressure, $\mu$ is the dynamic viscosity, $\rho_{0}$ is fluid density, $\kappa$ is thermal diffusivity of the fluid, $K$ is permeability of the porous medium, $\phi$ is porosity of the porous medium, $S$ is ratio of heat capacities of the fluid in porous medium to the fluid in fluid layer,

The dimensionless equations w.r.t perturbed variables is

$\left[\frac{1}{\rho_{a}} \frac{\partial}{\partial t}-\nabla^{2}\right] \nabla^{2} w=0$

$\left[\frac{\partial}{\partial t}-\nabla^{2}\right] \theta=w$

$\left[\frac{D_{a}}{P_{r_{n}}} \frac{\partial}{\partial t}+1\right] \nabla_{n}^{2} w_{n}=0$

$\left[S \frac{\partial}{\partial t}-\nabla_{n}^{2}\right] \theta_{n}=w_{n}$

For the fluid layer, $\operatorname{Pr}=\frac{v}{\kappa}$ is the Prandtl number and for the Porous layer $\operatorname{Pr}_{n}=\frac{\mathrm{v}}{\kappa_{n} \phi}$ is the Prandtl number, and $D a=\frac{K}{d_{n}{ }^{2}}$ is the Darcy number. The set of equations (7)-(10) can be solved by employing boundary conditions with upper and lower boundaries.

$W_{i}=\frac{1}{2}\left(2 z^{4}-5 z^{3}+3 z^{2}\right) T_{i-1}$

$\Theta_{i}=\frac{z}{2}(2-z) T_{i-1}$

$\Phi_{i}=\frac{z^{2}}{3}(3-2 z) T_{i-1}$

Here $T_{i-1}$ indicates modified chebyshev polynomial.

The trial solution obtained here satisfies all the prescribed boundary conditions, with an exception, namely $D \Theta+B_{i} \Theta=0$ at $z=1$. In this the residual is been included in the D.E as a Residual term.

The prescribed boundary conditions are

$W=D W=\Phi=D \Theta=0 \quad$ (14) on the lower rigid boundary, $W=D W=\Phi=D \Theta+B_{i} \Theta=0(15)$ on the upper rigid boundary and

$W=D^{2} W=D \Phi=D \Theta+B_{i} \Theta=0(16)$ on the upper boundary is free.

\section{METHOD OF SOLUTION}

Equations (14) to (16) along with the natural boundary condition comprise the eigen value problem. The problem can be solved using Galerkin method.

Let us define basis functions as

$$
\begin{aligned}
& W(z)=\sum_{i=1}^{n} \alpha_{i} W_{i}(z) \\
& \Theta(z)=\sum_{i=1}^{n} \beta_{i} \Theta_{i}(z) \\
& \Phi(z)=\sum_{i=1}^{n} \gamma_{i} \Phi_{i}(z)
\end{aligned}
$$

the constants $\alpha_{i}, \beta_{i}$ and $\gamma_{i}$ are to be found.

Basis functions are selected such that satisfy corresponding B.C's.

The trial functions for rigid-rigid boundaries are given by

$$
\begin{aligned}
& W_{i}=z^{2}\left(1-z^{2}\right) T_{i-1} \\
& \Theta_{i}=\frac{z}{2}(2-z) T_{i-1} \\
& \Phi_{i}=z(1-z) T_{i-1}
\end{aligned}
$$

and for rigid tree boundaries, the trial functions are

$$
\begin{aligned}
& W_{i}=\left(z^{4}-2 z^{3}+z^{2}\right) T_{i-1} \\
& \Theta_{i}=\frac{z^{2}}{3}(3-2 z) T_{i-1} \\
& \Phi_{i}=\frac{z}{2}(2-z) T_{i-1}
\end{aligned}
$$

where $T_{i}$ 's are modified chebyshev polynomials of II kind, such that the trial function of rigid-rigid, rigid-free boundaries $W_{i}, \Theta_{i}$ and $\Phi_{i}$ satisfy all the prescribed B.C's with a lone exception of one normal condition $B_{i} \Theta+D \Theta=0$ at the point $z=1$, the residual theorem is taken care by adding it in the differential equation

$$
\begin{gathered}
\left(D^{2}-a^{2}\right)\left[\Lambda\left(D^{2}-a^{2}\right)-D a^{-1}\right] W= \\
a^{2} R\left(a+M_{1}\right) \Theta-a^{2} R M_{1} D \Phi \\
\left(D^{2}-a^{2}\right) \Theta=-\left(1-M_{2} A\right) W \\
\left(D^{2}-a^{2} M_{3}\right) \Phi=D \Theta
\end{gathered}
$$

Multiplying equation (1) by $W_{i}(z),(2)$ by $\Theta_{i}(z),(3)$ by $\Phi_{i}(z)$ and integrating w.r.t $z$ from 
0 to 1 with the help of natural B.C's, we set up homogeneous algebraic equation.

A linear homogeneous algebraic equation will have a non-trivial solution, if the determinant of the coefficient matrix is zero. We obtain the relation with the following parameters

$$
\begin{aligned}
& \Lambda, R, M_{1}, M_{2}, M_{3}, a, D a^{-1}, B_{i} \\
& F\left(a, B_{i}, D a^{-1}, \Lambda, R, M_{1}, M_{2}, M_{3}\right)=0
\end{aligned}
$$

In non-dimensional form, $f(z)$ can be given and $f(z)=a+2(z-1) b+3(z-1)^{2} c$ Where the temperature gradient $f(z)$ is chosen, such that $\int_{0}^{1} f(z) d z=1$

On integration, we obtain $a-b+c=1$

we observe that $a=1, b=0, c=0$ given the classical linear state temperature distribution.

Analytical Solution by Regular Perturbation Technique In this section, we solve the Eigen Value problem using RPT, with the perturbation parameter ' $a$ '. The wave number $a<<1$, under perfectly insulated boundaries. We can say that $D \Theta=0$ at $z=0$ and $z=1$. Expanding the trial functions $W, \Theta$ and $\Phi$ in powers of $a^{2}$, we have

$$
(W, \Theta, \Phi)=\left(W_{0}, \Theta_{0}, \Phi_{0}\right)+a^{2}\left(W_{1}, \Theta_{1}, \Phi_{1}\right)+\ldots \ldots
$$

Put (29) in equation (26) to (28) along with the boundary condition and taking out the zeroth order terms, we get

$$
\begin{gathered}
D^{4} W_{0}-D^{-1} a_{1} D^{2} W_{0}=0 \\
D^{2} \Theta_{0}=-W_{0} \\
D^{2} \Phi_{0}=D \Theta_{0}
\end{gathered}
$$

where $D^{-1} a_{1}=\frac{D^{-1} a}{\sqrt{\Lambda}}$ along with boundary conditions at $z=0$ as

$$
\begin{aligned}
& W_{0}=D W_{0}=0 \\
& D \Theta_{0}=0 \\
& \Phi_{0}=0
\end{aligned}
$$

The general solution obtained is $W_{0}=0, \Theta_{0}=0, \Phi_{0}=0$. Similarly, on taking out the first order terms, we get

$$
\begin{gathered}
D^{4} W_{1}-D^{2} D^{-1} a W_{1}=R_{1}\left(1+M_{1}\right) \\
D^{2} \Theta_{1}=1-W_{1} \\
D^{2} \Phi_{1}=D \Theta_{1}
\end{gathered}
$$

We have $R_{1}=\frac{R}{\Lambda}$ and B.C's

$$
\begin{aligned}
& W_{1}=D W_{1}=0 \\
& D \Theta_{1}=0
\end{aligned}
$$

$$
\Phi_{1}=0
$$

The general solution obtained is $W_{1}=c_{1}+c_{2} z+c_{3} \cosh \left(\sigma_{1} z\right)+$

$$
c_{4} \sinh \left(\sigma_{1} z\right)-\frac{R_{1}\left(1+M_{1}\right)}{2 \sigma_{1}^{2}} z^{2}
$$

\section{CONCLUSION}

V.

We can conclude from the exact solution and numerical solution that the Marangoni convection can be controlled effectively in a composite fluid in fluid and Porous layer by appropriately choosing the values of $\operatorname{Pr}, D a$ and $\alpha$.The ferro convection is been controlled mainly by viscosity of magnetic field. The latent values obtained here by regular perturbation technique and Galerkin method are alike and complement each other further strengthening the fact that analytical solutions obtained are near exact.

\section{REFERENCES}

[1] J.R.A. Pearson, on convection cells induced by surface tension, J.Fluid Mech.4 91958), pp. 489.

[2] L Rayleigh, on convection currents in a horizontal layer of fluid when the higher temperature is on the other side, Phil.Mag.32 (1916), pp. 529.

[3] C.E Nanjundappa, and I.S. Shivakumara, Effect of velocity and temperature boundary conditions on convective instability in a ferrofluid layer, ASME J. Heat transf.130 (2008), 1045021-1045025.

[4] C.E Nanjundappa, I.S. Shivakumara, and K.Srikumar, Effect of MFDviscosity on the onset of ferromagnetic fluids layer heated from below and cooled from above with constant heat flux, Meas.Sci.Rev.9(3) (2009), 77-78.

[5] I.S. Shivakumara, J.Lee and C.E Nanjundappa, Onset of thermogravitational convection in a ferrofluid layer with temperature dependent viscosity, ASME J. Heat transf.134 (2012), 0125011-0125017.

[6] I.S. Shivakumara, N.Rudraiah and C.E Nanjundappa, Effect of non-uniform basic temperature gradient on Rayleigh-Benard-Marangoni convection in ferrofluids, J.Magn.Magn.Mater.248, 379-395.

[7] I.S. Shivakumara and C.E Nanjundappa, Marangoni ferroconvection with different initial temperature gradients, J.Energy Heat Mass Transf.28, 45-61.

[8] C.E Nanjundappa, I.S. Shivakumara, and R. Arunkumar, Benard-Marangoni ferroconvection with magnetic field dependent viscosity, J.Magn.Magn.Mater.322, 2256-2263.

[9] C.E Nanjundappa, I.S. Shivakumara, and R. Arunkumar, Onset of Marangoni-Benard ferro-convection with temperature dependent viscosity, Microgravity Sci.Technol.25(2013), 103-112.

[10] C.E Nanjundappa, I.S. Shivakumara, and B.Savitha, Onset of Benard-Marangoni ferroconvection with a convective surface boundary condition: The effects of cubic temperature profile 
and MFD viscosity, International Communications in Heat and Mass Transfer, 51, 39-44.

[11] C.E Nanjundappa, H.N.Prakash, I.S. Shivakumara and Jinho Lee, Effect of temperature dependent viscosity on the onset of Benard-Marangoni ferroconvection, International Communications in Heat and Mass Transfer, 51, 25-30.

[12] C.E Nanjundappa, I.S. Shivakumara and Jinho Lee, Effect of Coriolis force on Benard-Marangoni convection in a rotating ferrofluid layer with MFD viscosity, Micrgavity Sci.Technol, Vol27, 27-37. 